\title{
A foot-drop case
}

\author{
Daniela Columpsi - Matteo Badini - Emanuela Scannella • \\ Nicola Montano $\cdot$ Marco Antivalle $\cdot$ Massimo Tonolini • \\ Maurizio Osio
}

Received: 17 March 2010/Accepted: 7 April 2010/Published online: 4 May 2010

(C) SIMI 2010

\section{Case presentation}

Dr. Montano: A 42-year-old man was admitted to our department because of migratory arthromyalgias predominantly involving large joints, with painful swelling for the prior 3 weeks. He had used large doses of non-steroid antiinflammatory drugs with limited pain control.

The patient was eupneic and apyretic demonstrating pain and swelling of the left hand, and erythema and edema of the right leg and foot. Blood pressure and heart rate were, respectively, $120 / 80 \mathrm{mmHg}$ and 80 beats/min.

The remaining clinical history and physical examination were otherwise negative.

The electrocardiogram and the chest X-ray were normal. Compressive ultrasound scan of the legs, looking for a deep venous thrombosis, was normal.

This case record was selected for the "Casi Clinici Gymnasium" session at the 2009 SIMI Annual Congress in Rome, and was reviewed by a Committee composed of Maria Domenica Cappellini and Luigi Pagliaro.

D. Columpsi $(\bowtie) \cdot$ M. Badini · E. Scannella $\cdot$ N. Montano Medicina Interna II, Ospedale L. Sacco, Università Degli Studi Di Milano, Milan, Italy

e-mail: daniela.columpsi@fastwebnet.it

M. Antivalle

Reumatologia, Ospedale L. Sacco,

Università Degli Studi di Milano, Milan, Italy

M. Tonolini

Radiologia, Ospedale L. Sacco, Università degli Studi di Milano, Milan, Italy

M. Osio

Neurologia, Ospedale L. Sacco, Università degli Studi di Milano, Milan, Italy
Routine laboratory findings were within the normal ranges except for hypertransaminasemia (AST 287, ALT 740); for this reason an abdominal ultrasound scan was performed, but showed only a mild hepatosplenomegaly. Serologic profile revealed previous Epstein-Barr and cytomegalovirus infections, and detected hepatitis $B$ virus (HBV) IgM antibodies, HBsAg and HBeAg, thus showing an acute $\mathrm{HBV}$ infection. Autoimmune screening (ANCA and ANA autoantibodies, rheumatoid factor, autoantibodies against gliadin and transglutaminase) were all normal as were the thyroid function tests.

The migratory arthralgias were treated with tramadol, with a significant reduction in pain.

Intravenous fluids were administered, and a specific diet for hepatic disease was prescribed; the patient was subsequently discharged with the diagnosis of "migratory arthralgias in an acute hepatitis B infection".

A month after discharge, the patient was readmitted to our department for dysesthesias and hypoesthesia of both feet (predominantly the right) associated with motor deficits.

On neurologic examination, flexion, extension and rotation movements of the right foot were not possible for the patient, and the Achilles tendon reflex was absent. The patient also showed a deficit in right thigh flexion with steppage gait, and a deficit in left foot extension.

Laboratory findings showed a leukocytosis (12,000 WBC) and C-reactive protein (CRP) elevation (170 mg/l).

A magnetic resonance (MR) examination of the spine was performed to test the hypothesis of spondylo-diskitis or multiple discopathies, but was normal.

To evaluate the motor deficit, an electromyography (EMG) was performed. It revealed an acute axonal multineuropathy involving the right common peroneal nerve and the left femoral nerve, associated with chronic axonal damage in some of the studied districts. In particular, 
because EMG detected a possible conduction block of the left tibial nerve, to exclude the existence of a pseudo conduction block, a subsequent control was programmed.

Then, hospital course was complicated by an episode of abdominal pain associated with increasing lipases, treated with antispasmodics with a significant reduction in symptoms. Moreover, the blood pressure over $24 \mathrm{~h}$ was continuously high for both systolic and diastolic arterial pressure, with a non-dipper profile, and not responding to therapy. Laboratory tests showed hypokalemia, proteinuria, and elevation of inflammatory indexes, associated with high renin and aldosterone plasmatic levels (renin 150.9 $\mathrm{pg} / \mathrm{mL}$, aldosterone $556 \mathrm{pg} / \mathrm{ml}$ ), thus suggesting a secondary hyperaldosteronism.

In addition, the patient reported an episode of diplopia of the right eye, associated with blurred vision: a cranial computed tomography (CT) scan performed was normal.

\section{Differential diagnosis}

Dr. Columpsi, Dr. Badini and Dr. Scannella: All our findings are suggestive for a multisystemic disease. In particular neuropathy, abdominal pain, newly diagnosed hypertension, a transient episode of diplopia, and migratory arthralgias are typical findings of different clinical pictures, such as vasculitis, connective tissue disease, neoplasia, amyloidosis, thromboembolism, or fibromuscular disease. Further studies and a rheumatology consultation are needed to better clarify the clinical picture.

\section{Preliminary diagnosis}

Dr. Antivalle (rheumatologist): I agree that in our patient the systemic symptoms and signs suggest a vasculitic process either primary or associated with a connective tissue disease. Moreover, the clinical picture in association with a hepatitis B infection and multiplex mononeuropathy strongly suggest polyarteritis nodosa (PAN) as the most likely diagnosis.

To confirm this preliminary diagnosis, it would be necessary to perform an arteriography or a computed tomography (CT scan) of the abdominal aorta, a second electromyography and a tissue biopsy of a clinically affected organ.

Moreover, laboratory analysis including assays for autoantibodies-neutrophil anticytoplasm (ANCA), antinuclear (ANA), double-stranded DNA (anti-DNA), extractable nuclear antigens (ENA)-rheumatoid factor, cryoglobulins and complement components (C3, C4) should be repeated to narrow the differential diagnosis.

\section{Further investigations}

Dr. Tonolini (radiologist): Multidetector CT angiography of the abdominal aorta shows multiple abnormalities of the visceral arteries, with a "string-of-beads" pattern of irregular constrictions and dilatations involving the gastroduodenal artery and the distal portions and intraparenchymal branches of both renal arteries, leading to a bilaterally impaired renal perfusion (Fig. 1). Coexisting features of an elongated, tortuous splenic artery and discrete caliber irregularities of the superior mesenteric artery are also of note (Fig. 2).

Moreover, the CT scan depicts a subacute pancreatitis of the body and tail probably due to an ischemic disease, with edema and peri-pancreatic fluid. This findings are suggestive of a vasculitis involving abdominal vessels.

Dr. Osio (neurologist): The second electromyography, performed 2 weeks later, reveals a progression of the previously observed multineuropathy, with ongoing denervation
Fig. 1 Multidetector CT angiography of the abdominal aorta: multiple microaneurysms and irregular constrictions ("string-of-beads" pattern) involving the distal and intraparenchymal portion of the renal arteries bilaterally, including the accessory polar artery on the left side. Bilaterally altered parenchymal contrast enhancement with multiple wedge-shaped hypoperfused areas in both kidneys
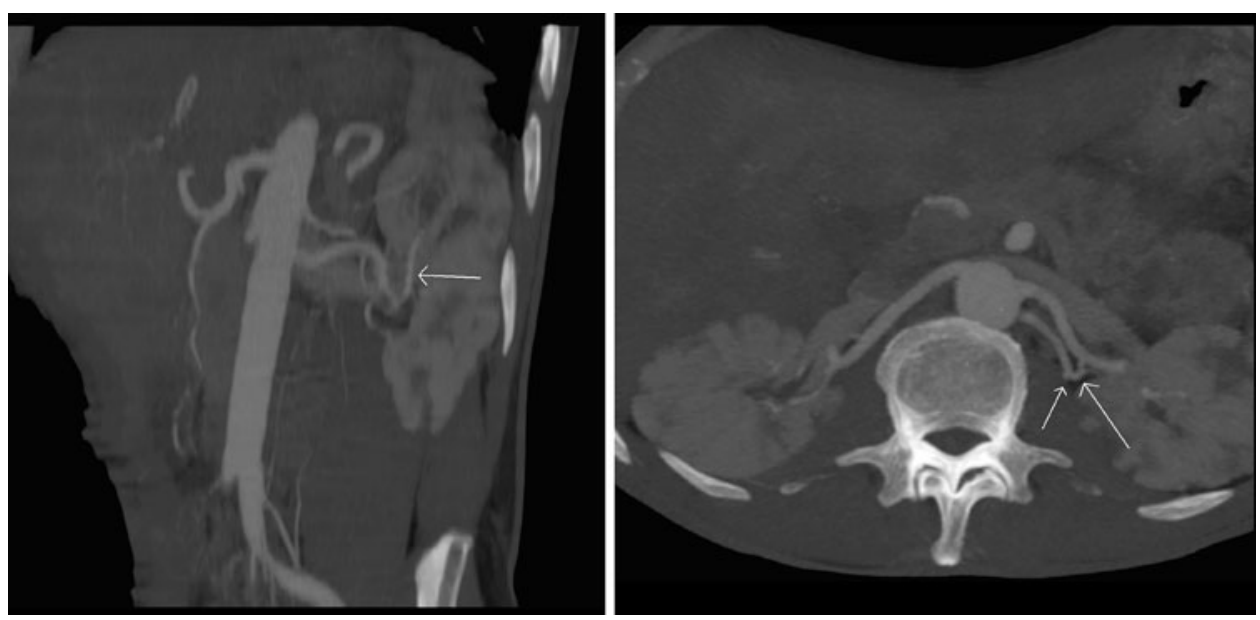


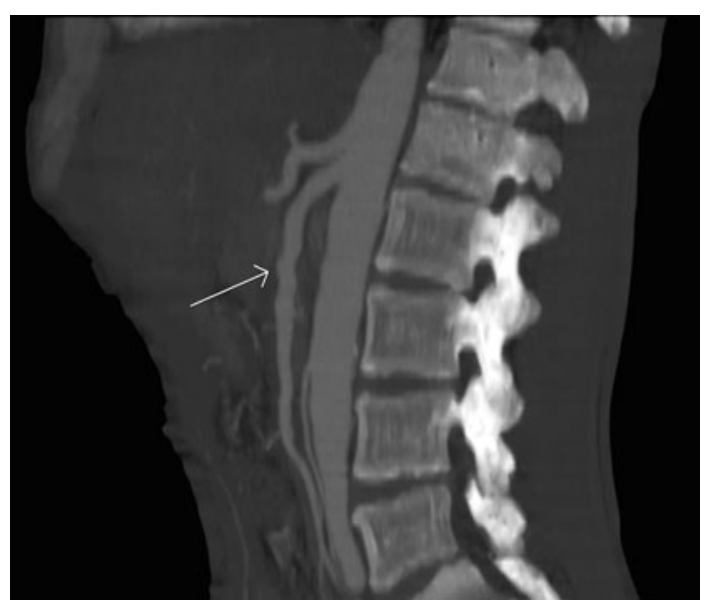

Fig. 2 Multidetector CT angiography of the abdominal aorta: diffuse, discrete calibre irregularity of the entire descending portion of the superior mesenteric artery

in a context of chronic axonal damage, and confirms the existence of a pseudo conduction block of the left tibial nerve. These findings are strongly suggestive of a vasculitic neuropathy.

Finally, the sural nerve biopsy reveals a chronic axonal neuropathy with ongoing axonal degeneration probably on a vasculitic basis. This pattern, associated with HBV infection, suggests the diagnosis of polyarteritis nodosa (PAN).

\section{Diagnosis and therapy}

Dr. Montano: The findings of previous HBV infection, mononeuropathy multiplex, and wandering arthromyalgias, the typical string-of-beads pattern demonstrated by the CT scan of the abdominal aorta and the sural nerve biopsy, met ACR (American College of Rheumatology) criteria for PAN [1].

The clinical picture can be explained by a multiorgan vasculitic involvement.

In fact, the involvement of the renal arteries provoked impaired renal perfusion and activation of the reninangiotensin system, leading to resistant hypertension, which is a common finding in patients with PAN. The abdominal pain, associated with increasing lipases, and the CT scan findings were likely due to an acute pancreatitis, probably provoked by a pancreatic ischemia.

The transient episode of diplopia was probably determined by vasculitic involvement of the oculomotor nerves, and must be considered as a direct result of vasa-vasorum occlusion provoking an ischemic lesion of the nerves.

Consequently, a specific therapy for PAN with methylprednisolone $(1 \mathrm{mg} / \mathrm{kg}$ daily by intravenous injection for
3 days, subsequently tapered to $0.6 \mathrm{mg} / \mathrm{kg}$ by mouth) was started. Moreover, an antiviral therapy with lamivudine was also started to treat acute hepatitis.

Dr. Columpsi, Dr. Badini and Dr. Scannella: Autoimmune screening, repeated after the rheumatologist's consultation, was again normal, and complement components also were in the normal range. In fact, auto antibodies rarely appear in patients with PAN.

How could we have suspected a vasculitis on the basis of the EMG findings?

Dr. Osio (neurologist): On the basis of our EMG findings, a diagnosis of multineuropathy of vasculitic origin could be suspected. Indeed, the presence of pseudo conduction block, due to axonal damage, in a pattern of mononeuropathy multiplex is strongly suggestive for a systemic vasculitic process affecting the vasa nervorum and thus causing multiple nerve infarcts. Because ischemic lesions accumulate randomly along the course of peripheral nerves, the clinical result is a pattern of damage to both sensory and motor nerves, initially asymmetric.

\section{Discussion}

Dr. Montano, Dr. Columpsi, Dr. Badini and Dr. Scannella: Polyarteritis nodosa is a systemic necrotizing vasculitis predominantly involving medium-sized muscular arteries. It is characterized by segmental vascular lesion involving the bifurcations and branches of the arteries.

Year after year, the frequency of HBV-PAN has declined from $35 \%$ in 1984 to $1-5 \%$ till today [2].

The vascular inflammation is probably due to deposition of an immune complex (composed of HBsAg and immunoglobulins) [3] inducing inflammation and fibrinoid necrosis, followed by thrombosis, infarction, and, sometimes, hemorrhage [4].

The prevalence of PAN following HBV acute infection is around 1-5\% [2] and usually becomes manifest $<12$ months after viral infection [5].

PAN can virtually affect any organ. The most common manifestations are fever, subacute weight loss, asthenia, renal involvement, hypertension, peripheral neuropathy, musculoskeletal and cutaneous involvement, cardiac failure, and gastrointestinal tract involvement.

A mononeuropathy multiplex, characterized by motor and sensory deficits, is one of the most common findings in patients with PAN, occurring in up to $60 \%$ of patients. The longest nerves in the body are primarily affected, and for this reason, a foot-drop is the most common manifestation, and it is also evident through observation of the patient during ambulation. 
For these reasons, in a patient with $\mathrm{HBV}$ infection and a clinical picture suggestive for a mononeuropathy multiplex, an electromyography should always be performed.

The prognosis of untreated PAN is relatively poor: the 5 -year survival is $13 \%$ [6, 7]. Renal failure and mesenteric, cardiac, or cerebral infarction are the major causes of death. Early detection is a key prerequisite for reducing mortality and morbidity of PAN due to the substantial decrease in complications that can be obtained if therapy is promptly introduced.

Therapy has improved the outcome to approximately 75-80\% survival at 5 years [7]. The treatment consists of the combination of high doses of glucocorticoids and a cytotoxic agent (usually cyclophosphamide) to achieve a remission characterized by an absence of active vascular inflammation [8].

Conflict of interest None.

\section{References}

1. Lightfoot RW Jr, Michel BA, Bloch DA et al (1990) The American College of Rheumatology 1990 criteria for the classification of polyarteritis nodosa. Arthritis Rheum 33:1088-1093

2. Pagnoux C, Cohen P, Guillevin L (2006) Vasculitides secondary to infections. Clin Exp Rheumatol 24:S71-S81

3. Guillevin L, Lhote F, Cohen P et al (1995) Polyarteritis nodosa related to hepatitis B virus. A prospective study with long-term observation of 41 patients. Medicine (Baltimore) 74:238-253

4. Chi ZC, Ma SZ (2003) Rheumatologic manifestations of hepatic diseases. Hepatobiliary Pancreat Dis Int 2:32-37

5. Guillevin L, Mahr A, Callard P et al (2005) Hepatitis B virusassociated polyarteritis nodosa: clinical characteristics, outcome, and impact of treatment in 115 patients. Medicine (Baltimore) 84:313-322

6. Balow JE (1985) Renal vasculitis. Kidney Int 27:954-964

7. Gayraud M, Guillevin L, le Toumelin P et al (2001) Long-term follow up of polyarteritis nodosa, microscopic polyangiitis, and Churg-Strauss syndrome. Arthritis Rheum 44:666-675

8. Leib ES, Restivo C, Paulus HE (1979) Immunosuppressive and corticosteroid therapy of polyarteritis nodosa. Am J Med 67:941-947 